\title{
Iterating the Sum-of-Divisors Function
}

\author{
Graeme L. Cohen and Herman J. J. te Riele
}

\section{CONTENTS}

\section{Introduction}

2. Tables of $(\mathrm{m}, \mathrm{k})$-Perfect Numbers

3. Is Every Number $(m, k)$-Perfect ?

4. Discussion of the Six Statements Acknowledgments

References
Let $\sigma^{0}(n)=n$ and $\sigma^{m}(n)=\sigma\left(\sigma^{m-1}(n)\right)$, where $m \geq 1$ and $\sigma$ is the sum-of-divisors function. We say that $n$ is $(m, k)$ perfect if $\sigma^{m}(n)=k n$. We have tabulated all $(2, k)$-perfect numbers up to $10^{9}$ and all $(3, k)$ - and $(4, k)$-perfect numbers up to $2 \cdot 10^{8}$. These tables have suggested several conjectures, some of which we prove here. We ask in particular: For any fixed $m \geq 1$, are there infinitely many $(m, k)$-perfect numbers? Is every positive integer $(m, k)$-perfect, for sufficiently large $m \geq 1$ ? In this connection, we have obtained the smallest value of $m$ such that $n$ is $(m, k)$-perfect, for $1 \leq n \leq 1000$. We also address questions concerning the limiting behaviour of $\sigma^{m+1}(n) / \sigma^{m}(n)$ and $\left(\sigma^{m}(n)\right)^{1 / m}$, as $m \rightarrow \infty$.

\section{INTRODUCTION}

All roman letters in this article denote positive integers, unless indicated otherwise, and $\sigma$ denotes the sum-of-divisors function.

There is a great deal of literature concerning the iteration of the function $\sigma(n)-n$, much of it concerned with whether the iterated values eventually terminate at zero, cycle or become unbounded, depending on the value of $n$. See [Erdös et al. 1990; Guy 1994, p. 62] for details.

Less work has been done on iterates of $\sigma$ itself. We define $\sigma^{0}(n)=n$ and $\sigma^{m}(n)=\sigma\left(\sigma^{m-1}(n)\right)$ for $m \geq 1$, and we call $n(m, k)$-perfect if $\sigma^{m}(n)=k n$. The classical perfect numbers are $(1,2)$-perfect. Multiperfect numbers are $(1, k)$-perfect, superperfect numbers are $(2,2)$-perfect, multiply superperfect numbers [Pomerance 1975] are $(2, k)$-perfect, m-superperfect numbers (ascribed by [Guy 1994, p. 65] to Bode; see also [Lord 1975]) are $(m, 2)-$ perfect.

Write $N_{p}=2^{p-1}$ when $2^{p}-1$ is a (Mersenne) prime. Superperfect numbers were introduced by Suryanarayana [1969], who showed there that the 
only even ones are the powers $N_{p}$. Bode and Lord, mentioned above, showed independently that an $m$-superperfect number can be even only if $m=2$.

For a simple proof of these facts, we note that, since $\sigma(n)=n \sum_{d \mid n}(1 / d)$, we have

$$
\sigma(\sigma(n))=n \sum_{d \mid n} \frac{1}{d} \sum_{e \mid \sigma(n)} \frac{1}{e} .
$$

Suppose $n$ is $m$-superperfect and $2^{a} \| n$ (that is, $2^{a} \mid n$ but $\left.2^{a+1} \nmid n\right)$. Then, for $m \geq 2$,

$$
\begin{aligned}
2 & =\frac{\sigma^{m}(n)}{n} \geq \frac{\sigma(\sigma(n))}{n} \\
& \geq\left(1+\frac{1}{2}+\cdots+\frac{1}{2^{a}}\right)\left(1+\frac{1}{2^{a+1}-1}\right)=2 .
\end{aligned}
$$

So as not to have a contradiction, we must have equality throughout. Thus, $m=2, n=2^{a}$ and $2^{a+1}-1$ is prime.

Kanold [1969] showed that an odd superperfect number must be a perfect square. This is similarly proved, using (1.1). For suppose $n$ is superperfect, and that $\sigma(n)$ is even. Say $2^{a} \| \sigma(n)$, so that $\left(2^{a+1}-1\right) \mid n$ since $n$ is superperfect. Then

$2=\frac{\sigma(\sigma(n))}{n} \geq\left(1+\frac{1}{2^{a+1}-1}\right)\left(1+\frac{1}{2}+\cdots+\frac{1}{2^{a}}\right)=2$.

Since we must have equality, we have both $\sigma(n)=$ $2^{a}$ and $n=2^{a+1}-1$. This contradiction means that $\sigma(n)$ must be odd, so, if $n$ is odd, then $n$ is a square.

Other work on the iteration of $\sigma$ has concerned whether

$$
s_{m}=\liminf _{n \rightarrow \infty} \frac{\sigma^{m}(n)}{n}
$$

is finite or not. See [Maier 1984], where $s_{3}$ is shown to be finite, and for the history of this problem.

In this paper, we will give particular attention to some questions raised in [Erdős et al. 1990]. The authors list the following six statements (reproduced in [Guy 1994, pp. 97-98]), with the comment: "We can neither prove nor disprove any of these statements." (i) For any $n>1, \sigma^{m+1}(n) / \sigma^{m}(n) \rightarrow 1$ as $m \rightarrow \infty$.

(ii) For any $n>1, \sigma^{m+1}(n) / \sigma^{m}(n) \rightarrow \infty$ as $m \rightarrow \infty$.

(iii) For any $n>1,\left(\sigma^{m}(n)\right)^{1 / m} \rightarrow \infty$ as $m \rightarrow \infty$.

(iv) For any $n>1$, there is $m$ with $n \mid \sigma^{m}(n)$.

(v) For any $n, l>1$, there is $m$ with $l \mid \sigma^{m}(n)$.

(vi) For any $n_{1}, n_{2}>1$, there are $m_{1}, m_{2}$ with $\sigma^{m_{1}}\left(n_{1}\right)=\sigma^{m_{2}}\left(n_{2}\right)$.

We will give some computational evidence to indicate that statements (ii), (iii), (iv) and (v) are true, and that statements (i) and (vi) are false.

Hausman [1982] has considered questions corresponding to some of those here for the Euler phifunction. In particular, she has completely characterised all $n$ such that $n=k \varphi^{m}(n)$, where $\varphi^{m}$ is defined analogously to $\sigma^{m}$.

\section{TABLES OF $(m, k)$-PERFECT NUMBERS}

Table 1 gives all $(2, k)$-perfect numbers up to $10^{9}$. In [Cohen and te Riele 1995], we also give all $(3, k)$ and $(4, k)$-perfect numbers up to $2 \cdot 10^{8}$. They are given in terms of increasing values of $k$. Corresponding lists, given as originally obtained with $n$ increasing, are available from the authors. All the following comments arise from inspections of such lists.

Many conjectures can be made, along the lines of that in [Guy 1994, p. 48] that there are only finitely many $(1, k)$-perfect numbers for $k \geq 3$. That particular conjecture is well-supported by the list that has been accumulated by [Schroeppel 1993], showing over 2000 such numbers, which is almost three times the number that were known just three years ago, and especially by the facts that no new $(1,3)$ perfect numbers have been found in the last 350 years, nor any new $(1,4)$-perfect numbers in the last 65 years. On the other hand, if the well-known conjecture that there are infinitely many powers $N_{p}$ is true, there are infinitely many $(1,2)$-perfect numbers.

There is a parallel situation with $(2, k)$-perfect numbers, of which there are families involving the powers $N_{p}$. Besides the well-known result that $N_{p}$ is $(2,2)$-perfect, we know that: 


\begin{tabular}{|c|c|c|c|c|c|}
\hline$k$ & $n$ & $k$ & $n$ & $k$ & $n$ \\
\hline 1 & 1 & 8 & $960=2^{6} \cdot 3 \cdot 5$ & & $4404480=2^{8} \cdot 3 \cdot 5 \cdot 31 \cdot 37$ \\
\hline 2 & $2=2$ & 8 & $4092=2^{2} \cdot 3 \cdot 11 \cdot 31$ & 11 & $57669920=2^{5} \cdot 5 \cdot 7 \cdot 11 \cdot 31 \cdot 151$ \\
\hline 2 & $4=2^{2}$ & 8 & $16368=2^{4} \cdot 3 \cdot 11 \cdot 31$ & 11 & $238608384=2^{13} \cdot 3 \cdot 7 \cdot 19 \cdot 73$ \\
\hline 2 & $16=2^{4}$ & 8 & $58254=2 \cdot 3 \cdot 7 \cdot 19 \cdot 73$ & 12 & $2200380=2^{2} \cdot 3 \cdot 5 \cdot 7 \cdot 13^{2} \cdot 31$ \\
\hline 2 & $64=2^{6}$ & 8 & $61440=2^{12} \cdot 3 \cdot 5$ & 12 & $8801520=2^{4} \cdot 3 \cdot 5 \cdot 7 \cdot 13^{2} \cdot 31$ \\
\hline 2 & $4096=2^{12}$ & 8 & $65472=2^{6} \cdot 3 \cdot 11 \cdot 31$ & 12 & $14913024=2^{9} \cdot 3 \cdot 7 \cdot 19 \cdot 73$ \\
\hline 2 & $65536=2^{16}$ & 8 & $116508=2^{2} \cdot 3 \cdot 7 \cdot 19 \cdot 73$ & 12 & $35206080=2^{6} \cdot 3 \cdot 5 \cdot 7 \cdot 13^{2} \cdot 31$ \\
\hline 2 & $262144=2^{18}$ & 8 & $466032=2^{4} \cdot 3 \cdot 7 \cdot 19 \cdot 73$ & 12 & $140896000=2^{8} \cdot 5^{3} \cdot 7 \cdot 17 \cdot 37$ \\
\hline 3 & $8=2^{3}$ & 8 & $710400=2^{8} \cdot 3 \cdot 5^{2} \cdot 37$ & 12 & $459818240=2^{8} \cdot 5 \cdot 7 \cdot 19 \cdot 37 \cdot 73$ \\
\hline 3 & $21=3 \cdot 7$ & 8 & $983040=2^{16} \cdot 3 \cdot 5$ & 12 & $775898880=2^{8} \cdot 3 \cdot 5 \cdot 37 \cdot 43 \cdot 127$ \\
\hline 3 & $512=2^{9}$ & 8 & $1864128=2^{6} \cdot 3 \cdot 7 \cdot 19 \cdot 73$ & 13 & $57120=2^{5} \cdot 3 \cdot 5 \cdot 7 \cdot 17$ \\
\hline 4 & $15=3 \cdot 5$ & 8 & $3932160=2^{18} \cdot 3 \cdot 5$ & 13 & $932064=2^{5} \cdot 3 \cdot 7 \cdot 19 \cdot 73$ \\
\hline 4 & $1023=3 \cdot 11 \cdot 31$ & 8 & $4190208=2^{12} \cdot 3 \cdot 11 \cdot 31$ & 13 & $3932040=2^{3} \cdot 3 \cdot 5 \cdot 7 \cdot 31 \cdot 151$ \\
\hline 4 & $29127=3 \cdot 7 \cdot 19 \cdot 73$ & 8 & $67043328=2^{16} \cdot 3 \cdot 11 \cdot 31$ & 13 & $251650560=2^{9} \cdot 3 \cdot 5 \cdot 7 \cdot 31 \cdot 151$ \\
\hline 6 & $42=2 \cdot 3 \cdot 7$ & 8 & $119304192=2^{12} \cdot 3 \cdot 7 \cdot 19 \cdot 73$ & 14 & $217728=2^{7} \cdot 3^{5} \cdot 7$ \\
\hline 6 & $84=2^{2} \cdot 3 \cdot 7$ & 8 & $268173312=2^{18} \cdot 3 \cdot 11 \cdot 31$ & 14 & $1278720=2^{8} \cdot 3^{3} \cdot 5 \cdot 37$ \\
\hline 6 & $160=2^{5} \cdot 5$ & 9 & $168=2^{3} \cdot 3 \cdot 7$ & 14 & $2983680=2^{8} \cdot 3^{2} \cdot 5 \cdot 7 \cdot 37$ \\
\hline 6 & $336=2^{4} \cdot 3 \cdot 7$ & 9 & $10752=2^{9} \cdot 3 \cdot 7$ & 14 & $5621760=2^{11} \cdot 3^{2} \cdot 5 \cdot 61$ \\
\hline 6 & $1344=2^{6} \cdot 3 \cdot 7$ & 9 & $331520=2^{8} \cdot 5 \cdot 7 \cdot 37$ & 14 & $14008320=2^{14} \cdot 3^{2} \cdot 5 \cdot 19$ \\
\hline 6 & $86016=2^{12} \cdot 3 \cdot 7$ & 9 & $691200=2^{10} \cdot 3^{3} \cdot 5^{2}$ & 14 & $298721280=2^{13} \cdot 3 \cdot 5 \cdot 11 \cdot 13 \cdot 17$ \\
\hline 6 & $550095=3 \cdot 5 \cdot 7 \cdot 13^{2} \cdot 31$ & 9 & $1556480=2^{14} \cdot 5 \cdot 19$ & 14 & $955367424=2^{14} \cdot 3^{2} \cdot 11 \cdot 19 \cdot 31$ \\
\hline 6 & $1376256=2^{16} \cdot 3 \cdot 7$ & 9 & $1612800=2^{10} \cdot 3^{2} \cdot 5^{2} \cdot 7$ & 15 & $1058148=2^{2} \cdot 3^{2} \cdot 7 \cdot 13 \cdot 17 \cdot 19$ \\
\hline 6 & $5505024=2^{18} \cdot 3 \cdot 7$ & 9 & $106151936=2^{14} \cdot 11 \cdot 19 \cdot 31$ & 15 & $29352960=2^{10} \cdot 3^{2} \cdot 5 \cdot 7^{2} \cdot 13$ \\
\hline 7 & $24=2^{3} \cdot 3$ & 10 & $480=2^{5} \cdot 3 \cdot 5$ & 16 & $7526400=2^{11} \cdot 3 \cdot 5^{2} \cdot 7^{2}$ \\
\hline 7 & $1536=2^{9} \cdot 3$ & 10 & $504=2^{3} \cdot 3^{2} \cdot 7$ & 16 & $23591520=2^{5} \cdot 3^{3} \cdot 5 \cdot 43 \cdot 127$ \\
\hline 7 & $47360=2^{8} \cdot 5 \cdot 37$ & 10 & $13824=2^{9} \cdot 3^{3}$ & 16 & $55046880=2^{5} \cdot 3^{2} \cdot 5 \cdot 7 \cdot 43 \cdot 127$ \\
\hline 7 & $343976=2^{3} \cdot 19 \cdot 31 \cdot 73$ & 10 & $32256=2^{9} \cdot 3^{2} \cdot 7$ & 18 & $39352320=2^{11} \cdot 3^{2} \cdot 5 \cdot 7 \cdot 61$ \\
\hline 8 & $60=2^{2} \cdot 3 \cdot 5$ & 10 & $32736=2^{5} \cdot 3 \cdot 11 \cdot 31$ & 19 & $312792480=2^{5} \cdot 3^{2} \cdot 5 \cdot 7^{2} \cdot 11 \cdot 13 \cdot 31$ \\
\hline 8 & $240=2^{4} \cdot 3 \cdot 5$ & 10 & $1980342=2 \cdot 3^{3} \cdot 7 \cdot 13^{2} \cdot 31$ & 22 & $83825280=2^{7} \cdot 3^{5} \cdot 5 \cdot 7^{2} \cdot 11$ \\
\hline
\end{tabular}

TABLE 1. All $(2, k)$-perfect numbers $n$ with $n<10^{9}$

(A) $N_{p} \cdot 3 \cdot 7$ is $(2,6)$-perfect.

(B) $N_{p} \cdot 3 \cdot 7 \cdot 19 \cdot 73$ is $(2,8)$-perfect; for $p>2, N_{p} \cdot 3 \cdot 5$ and $N_{p} \cdot 3 \cdot 11 \cdot 31$ are $(2,8)$-perfect.

(C) For $p>2, N_{p} \cdot 3 \cdot 5 \cdot 7 \cdot 13^{2} \cdot 31$ is $(2,12)$-perfect.

These are particular cases of the following general result.

Theorem 2.1. Suppose that $l$ is an odd $(2, k)$-perfect number. For any a such that $2^{a} \mid k \sigma\left(\sigma\left(2^{a}\right)\right)$ and such that $\sigma\left(2^{a}\right)$ and $\sigma(l)$ are relatively prime, the number $2^{a} l$ is $\left(2,2^{-a} k \sigma\left(\sigma\left(2^{a}\right)\right)\right)$-perfect.

Proof. Since $l$ is odd we have $\sigma\left(2^{a} l\right)=\sigma\left(2^{a}\right) \sigma(l)$, and since $\left(\sigma\left(2^{a}\right), \sigma(l)\right)=1$ we have

$$
\begin{aligned}
\sigma\left(\sigma\left(2^{a} l\right)\right) & =\sigma\left(\sigma\left(2^{a}\right)\right) \sigma(\sigma(l))=\sigma\left(\sigma\left(2^{a}\right)\right) k l \\
& =2^{-a} k \sigma\left(\sigma\left(2^{a}\right)\right) \cdot 2^{a} l .
\end{aligned}
$$

As a corollary, when $\sigma\left(2^{a}\right)$ is a (Mersenne) prime the condition $2^{a} \mid k \sigma\left(\sigma\left(2^{a}\right)\right)$ is true and, provided $\sigma\left(2^{a}\right) \nmid \sigma(l)$, the number $2^{a} l$ is $(2,2 k)$-perfect. The statements (A), (B) and (C) above all arise from an application of this theorem to the five nontrivial examples of odd $(2, k)$-perfect numbers in Table 1. Furthermore, we may, for example, apply the more general result of Theorem 2.1 to the $(2,4)$-perfect number $3 \cdot 7 \cdot 19 \cdot 73$, with $a=5,9,13$ (but to no other values of $a$ that we could find). In this way, we can deduce a family of $(2, k)$-perfect numbers 
(with varying $k$ ) that is "larger" than the set of Mersenne primes.

No other possibly infinite family of $(2, k)$-perfect numbers has been noticed, and we may conjecture that, apart from the above, there are only finitely many of these numbers for each $k$. We would also make the uncharacteristic conjecture that all $(2,4)$ perfect numbers are odd! Notice from Table 1 that we have found $(2, k)$-perfect numbers for all $k \leq 16$, except for $k=5$, and we conjecture that there are no $(2,5)$-perfect numbers.

No pattern has been discerned in $(m, k)$-perfect numbers, with any $m \geq 3$, and we conjecture that there are only finitely many for each $k$.

Some interrelationships between the tables have been noticed. The following facts, for example, are easily verified.

(D) If $n$ is $(2,4)$-perfect, $n$ is odd and $7 \nmid \sigma(n)$, then $n$ is $(4,32)$-perfect.

(E) If $n$ is $(2,7)$-perfect, $7 \nmid n$ and $2^{2} \| \sigma(n)$, then $n$ is $(4,63)$-perfect.

The next result can be contrasted with the easily proved result that the equation $\sigma(2 n)=2 \sigma(n)$ has no solutions.

Theorem 2.2. The equation $\sigma(\sigma(2 n))=2 \sigma(\sigma(n))$ has infinitely many solutions.

Proof. We need only verify that this equation is satisfied by $n=2 t$ for any $t$ with $(2, t)=(3, \sigma(t))=$ $(7, \sigma(t))=1$, and that any prime $t \equiv 1(\bmod 21)$ satisfies these conditions. There are infinitely many such primes.

This theorem can be generalised in various ways. For example, we have $\sigma\left(\sigma\left(2^{a} n\right)\right)=2^{a} \sigma(\sigma(n))$ when $n=2^{a} t$, where

$$
(2, t)=\left(2^{a+1}-1, \sigma(t)\right)=\left(2^{2 a+1}-1, \sigma(t)\right)=1
$$

and $2^{a+1}-1$ and $2^{2 a+1}-1$ are primes. The latter is the case for $a=1$ (as in the proof), and $a=2$, 6,30 .

\section{IS EVERY NUMBER $(\mathrm{m}, \mathrm{k})$-PERFECT?}

In support of statement (iv) from the Introduction, that all numbers $n$ are $(m, k)$-perfect for $m$ large enough, we have successfully tested all values of $n$ up to 1000 . In this connection, it is convenient to define

$$
\begin{aligned}
& \tilde{m}(n)=\inf \left\{m \geq 1: \frac{\sigma^{m}(n)}{n} \text { is an integer }\right\}, \\
& \tilde{k}(n)=\frac{\sigma^{\tilde{m}(n)}(n)}{n} .
\end{aligned}
$$

(If $\widetilde{m}(n)$ is infinite, we understand $\tilde{k}(n)$ to be infinite also.)

Representative values of $\widetilde{m}$ and $\tilde{k}$ are given in Table 2. A more complete version of this listing [Cohen and te Riele 1995, Table 4] gives the data for all $n \leq 400$.

We will comment on the more computationally difficult cases later; they tend to be those for which $\tilde{m}(n)>n$. There are fourteen such cases up to $n=400$, namely $n=3,11,29,53,58,59,67,101$, 109, 131, 149, 173, 202, 239.

The values of $\tilde{k}(n)$ of course become extremely large, the largest observed value in Table 2 being $\tilde{k}(389) \approx 5 \cdot 10^{232}$ and the largest for $n \leq 1000$ being $\tilde{k}(659) \approx 1.5 \cdot 10^{1183}$. It is interesting then that the following theorem allows us to predict exact values of $\widetilde{m}(n)$ and $\tilde{k}(n)$ in many cases, making use of earlier values.

Theorem 3.1. Suppose there are integers $n, t \geq 2, a$ and $M$ such that $\widetilde{m}(n)$ is finite, $t \mid \tilde{k}(n)$,

$$
\sigma^{M+a}(n)=\sigma^{M}(t n)
$$

and $M<\tilde{m}(n)-a$. Then $\tilde{m}(t n) \leq \widetilde{m}(n)-a$. If $\tilde{m}(t n)=\tilde{m}(n)-a$, then $\tilde{k}(t n)=\tilde{k}(n) / t$. If $\widetilde{m}(t n)<\widetilde{m}(n)-a$, then $\widetilde{m}(t n)<M$ and $\tilde{k}(t n)<$ $\alpha \tilde{k}(n) / t$, where

$$
\alpha=\frac{\sigma^{M+a}(n)}{\sigma^{\widetilde{m}(n)}(n)}<1
$$




\begin{tabular}{|c|c|c|c|c|c|c|c|c|c|c|c|}
\hline$n$ & $\tilde{m}$ & $\tilde{k}$ & & $n$ & $\tilde{m}$ & $\tilde{k}$ & & $n$ & $\tilde{m}$ & $\tilde{k}$ & \\
\hline 1 & 1 & 1 & & 55 & 19 & $8.2 \mathrm{E} 0082$ & $2^{24} 7^{2}$ & & & & \\
\hline 2 & 2 & 2 & 2 & 56 & 5 & 1822 & $2 \cdot 7 \cdot 13$ & 348 & 22 & $2.8 \mathrm{E} 011$ & $2^{9} 3.5$ \\
\hline 3 & 4 & 5 & 5 & 57 & 13 & 271852 & $2^{2} 7^{2} 19$ & 349 & 188 & $3.5 \mathrm{E} 140$ & $2^{86} 3^{29} 5^{7} 7^{8} 11^{4} 13^{2} 17^{3} 19^{7}$ \\
\hline 4 & 2 & 2 & 2 & 58 & 67 & $3.9 \mathrm{E} 042$ & $2^{21} 3^{2} 5^{2} 7^{2} 11 \cdot 19^{2}$ & 350 & 16 & $3.7 \mathrm{E} 007$ & $2^{7} 3^{6} 13 \ldots$ \\
\hline 5 & 5 & 24 & $2^{3} 3$ & 59 & 97 & $1.2 \mathrm{E} 064$ & $2^{56} 3^{6} 5 \cdot 7^{3} 11 \cdot 17 \cdot 19 \ldots$ & 351 & 19 & $1.7 \mathrm{E} 009$ & $2^{7} 3 \cdot 5 \cdot 11 \cdot 19 \ldots$ \\
\hline 6 & 1 & 2 & 2 & 60 & 2 & 82 & $2^{3}$ & 352 & 5 & 933 & $3 \ldots$ \\
\hline 7 & 5 & 24 & $2^{3} 3$ & 61 & 23 & $2.7 \mathrm{E} 011$ & $2^{10} 3^{3} 5 \cdot 7 \ldots$ & 353 & 263 & $1.4 \mathrm{E} 2012$ & $2^{74} 3^{27} 5^{13} 7^{10} 11^{3} 13^{4} 17 \cdot 19^{7}$ \\
\hline 8 & 2 & 3 & 3 & 62 & 5 & 962 & $2^{5} 3$ & 354 & 69 & $3.4 \mathrm{E} 0412$ & $2^{39} 3^{4} 7^{2} 11 \cdot 13 \cdot 19^{2} \ldots$ \\
\hline 9 & 7 & 168 & $2^{3} 3 \cdot 7$ & 63 & 16 & $5.7 \mathrm{E} 006$ & $2^{3} 3^{2} 5 \cdot 7 \cdot 11^{2} 19$ & 355 & 42 & $1.6 \mathrm{E} 0242$ & $2^{16} 3^{7} 5 \cdot 7^{3} 11 \cdot 19 \ldots$ \\
\hline 10 & 4 & 12 & $2^{2} 3$ & 64 & 2 & 22 & 2 & 356 & 9 & 95682 & $2^{5} 13 \ldots$ \\
\hline 11 & 15 & $1.8 \mathrm{E} 006$ & $2^{6} 3^{2} 5 \cdot 7^{2} 13$ & 65 & 4 & 242 & $2^{3} 3$ & 357 & 10 & 51202 & $2^{10} 5$ \\
\hline 12 & 3 & 10 & $2 \cdot 5$ & 66 & 8 & 1078 & $2 \cdot 7^{2} 11$ & 358 & 742 & $2.8 \mathrm{E} 0482$ & $2^{22} 3^{8} 5 \cdot 7^{4} 19^{3}$. \\
\hline 13 & 13 & 84480 & $2^{9} 3 \cdot 5 \cdot 11$ & 67 & 101 & $9.4 \mathrm{E} 066$ & $2^{21} 3^{10} 7^{4} 11 \cdot 13 \cdot 17 \cdot 19^{3}$. & 359 & 166 & $1.1 \mathrm{E} 1202$ & $2^{47} 3^{15} 5^{7} 7^{10} 11^{2} 13^{4} 17 \cdot 19^{7} \ldots$ \\
\hline 14 & 3 & 12 & $2^{2} 3$ & 68 & 21 & $4.6 \mathrm{E} 010$ & $2^{16} 3^{2} 5 \cdot 61 \ldots$ & 360 & 8 & 43691 & $17 \ldots$ \\
\hline 15 & 2 & 4 & $2^{2}$ & 69 & 19 & $3.2 \mathrm{E} 009$ & $2^{13} 7^{3} 13 \ldots$ & 361 & 19 & $1.5 \mathrm{E} 0082$ & $2^{7} 3^{2} 7^{2}$ \\
\hline 16 & 2 & 2 & 2 & 70 & 11 & 26624 & $2^{11} 13$ & 362 & 53 & $7.6 \mathrm{E} 0322$ & $2^{23} 3^{4} 5^{2} 7^{2} 11 \cdot 13 \cdot 19^{2}$ \\
\hline 17 & 13 & 92520 & $2^{3} 3^{2} 5$ & 71 & 50 & $8.0 \mathrm{E} 027$ & $2^{11} 3^{3} 5 \cdot 7^{5} 11 \cdot 13$ & 363 & 10 & 125442 & $2^{8} 7^{2}$ \\
\hline 18 & 4 & 20 & $2^{2} 5$ & 72 & 4 & 28 & $2^{2} 7$ & 364 & 13 & 5518802 & $2^{3} 3^{3} 5 \cdot 7 \ldots$ \\
\hline 19 & 12 & 62720 & $2^{8} 5 \cdot 7^{2}$ & 73 & 20 & $8.5 \mathrm{E} 008$ & $2^{9} 7^{2}$. & 365 & 42 & $1.7 \mathrm{E} 0242$ & $2^{15} 3^{6} 5^{3} 7^{2} 13 \cdot 17 \ldots$ \\
\hline 20 & 5 & 84 & $2^{2} 3 \cdot 7$ & 74 & 20 & $2.0 \mathrm{E} 009$ & $2^{6} 3^{2} 11 \cdot 19 \ldots$ & 366 & 15 & $1.0 \mathrm{E} 0072$ & $2^{10} 5 \ldots$ \\
\hline 21 & 2 & 3 & 3 & 75 & 23 & $5.6 \mathrm{E} 010$ & $2^{4} 3^{2} 17 \cdot 19 \ldots$ & 367 & 146 & $1.5 \mathrm{E} 1052$ & $2^{65} 3^{21} 5^{3} 7^{6} 11^{5} 13^{3} 17 \cdot 19^{4}$ \\
\hline 22 & 13 & 49920 & $2^{8} 3 \cdot 5 \cdot 13$ & 76 & 14 & $4.2 \mathrm{E} 006$ & $2^{4} 3 \cdot 7 \cdot 11 \ldots$ & 368 & 15 & $1.0 \mathrm{E} 0072$ & $2^{7} 3.5 \ldots$ \\
\hline 23 & 16 & $6.5 \mathrm{E} 006$ & $2^{9} 11 \cdot 13 \ldots$ & 77 & 21 & $4.5 \mathrm{E} 010$ & $2^{14} 3^{3} 5 \cdot 11^{2} 13^{2}$ & 369 & 35 & $5.1 \mathrm{E} 0202$ & $2^{18} 3^{4} 5^{2} 7 \ldots$ \\
\hline 24 & 2 & 7 & 7 & 78 & 10 & 14080 & $2^{8} 5 \cdot 11$ & 370 & 7 & 7682 & $2^{8} 3$ \\
\hline 25 & 17 & 881280 & $2^{7} 3^{4} 5 \cdot 17$ & 79 & 36 & $6.0 \mathrm{E} 018$ & $2^{9} 3^{5} 5^{2} 7^{3} 11 \cdot 13^{2} 19 \ldots$ & 371 & 34 & $4.1 \mathrm{E} 0182$ & $2^{15} 3^{2} 5 \cdot 7 \cdot 11 \cdot 13 \cdot 17 \ldots$ \\
\hline 26 & 4 & 28 & $2^{2} 7$ & 80 & 5 & 124 & $2^{2}$. & 372 & 7 & 1530 & $2 \cdot 3^{2} 5 \cdot 17$ \\
\hline 27 & 9 & 3360 & $2^{5} 3 \cdot 5 \cdot 7$ & 81 & 15 & $2.2 \mathrm{E} 006$ & $2^{3} 7 \cdot 13$ & 373 & 145 & 4.3E 103 & $2^{56} 3^{28} 5^{4} 7^{6} 11 \cdot 13^{4} 17 \cdot 19 \ldots$ \\
\hline 28 & 1 & 2 & 2 & 82 & 42 & $1.2 \mathrm{E} 024$ & $2^{16} 3^{6} 5^{4} 7^{3} 11 \cdot 13 \cdot 19^{2}$ & 374 & 32 & $2.2 \mathrm{E} 0172$ & $2^{13} 3^{3} 5 \ldots$ \\
\hline 29 & 78 & $5.1 \mathrm{E} 047$ & $2^{29} 3^{5} 5^{2} 7^{2} 13 \cdot 19 \ldots$ & 83 & 26 & $3.6 \mathrm{E} 012$ & $2^{13} 3^{6} 5 \cdot 13^{2} 17 \ldots$ & 375 & 25 & $5.0 \mathrm{E} 0122$ & $2^{19} 3^{2} 5 \cdot 7 \cdot 13^{2}$ \\
\hline 30 & 7 & 728 & $2^{3} 7 \cdot 13$ & 84 & 2 & 6 & $2 \cdot 3$ & 376 & 64 & $5.6 \mathrm{E} 0372$ & $2^{16} 3^{5} 5^{2} 7 \cdot 11 \cdot 13 \cdot 19 \ldots$ \\
\hline 31 & 10 & 912 & $2^{4} 3 \cdot 19$ & 85 & 36 & $2.1 \mathrm{E} 017$ & $2^{26} 3^{3} 5^{2} 7^{2} 19 \ldots$ & 377 & 68 & $9.3 \mathrm{E} 0432$ & $2^{40} 3^{5} 5^{4} 7^{4} 11 \cdot 13^{3} 19^{3}$ \\
\hline 32 & 4 & 18 & $2 \cdot 3^{2}$ & 86 & 17 & $1.7 \mathrm{E} 008$ & $2 \cdot 3^{3} 13 \ldots$ & 378 & 7 & 29122 & $2^{5} 7 \cdot 13$ \\
\hline 33 & 17 & $1.9 \mathrm{E} 007$ & $2^{13} 19 \ldots$ & 87 & 43 & $2.4 \mathrm{E} 023$ & $2^{20} 3 \cdot 5^{3} 7 \cdot 11 \cdot 13^{3}$. & 379 & 67 & $6.4 \mathrm{E} 0412$ & $2^{25} 3^{13} 5 \cdot 7^{2} 19^{2} \ldots$ \\
\hline 34 & 11 & 46260 & $2^{2} 3^{2} 5 \ldots$ & 88 & 8 & 4158 & $2 \cdot 3^{3} 7 \cdot 11$ & 380 & 15 & $4.3 \mathrm{E} 0072$ & $2^{8} 3^{3} 7^{2} \ldots$ \\
\hline 35 & 6 & 144 & $2^{4} 3^{2}$ & 89 & 13 & $6.1 \mathrm{E} 005$ & $2^{8} 3 \cdot 5 \cdot 7 \ldots$ & 381 & 9 & 30722 & $2^{10} 3$ \\
\hline 36 & 5 & 42 & $2 \cdot 3 \cdot 7$ & 90 & 7 & 1008 & $2^{4} 3^{2} 7$ & 382 & 99 & $8.3 \mathrm{E} 066$ & $2^{38} 3^{18} 5^{5} 7^{2} 11^{4} 13^{6} 17^{3} 19$. \\
\hline 37 & 28 & $3.0 \mathrm{E} 013$ & $2^{14} 5 \cdot 7 \cdot 11 \cdot 13 \ldots$ & 91 & 17 & $1.8 \mathrm{E} 007$ & $2^{13} 3^{3} 5 \cdot 17$ & 383 & 250 & $9.4 \mathrm{E} 191$ & $2^{88} 3^{24} 5^{13} 7^{10} 11^{2} 13^{4} 17^{2} 19^{3} \ldots$ \\
\hline 38 & 22 & $3.8 \mathrm{E} 010$ & $2^{5} 3^{4} 5 \cdot 7^{2} 13 \ldots$ & 92 & 14 & $1.6 \mathrm{E} 006$ & $2^{7} 11 \cdot 13 \ldots$ & 384 & 6 & 3411 & $11 \ldots$ \\
\hline 39 & 4 & 30 & $2 \cdot 3 \cdot 5$ & 93 & 10 & 5824 & $2^{6} 7 \cdot 13$ & 385 & 14 & 9480242 & $2^{3} 3^{4} 7 \cdot 11 \cdot 19$ \\
\hline 40 & 7 & 663 & $3 \cdot 13 \cdot 17$ & 94 & 54 & $5.8 \mathrm{E} 031$ & $2^{39} 3^{2} 5 \cdot 7 \cdot 11^{2} 19 \ldots$ & 386 & 81 & $4.3 \mathrm{E} 0532$ & $2^{33} 3^{6} 5^{4} 7^{5} 13 \cdot 17^{2} 19 \ldots$ \\
\hline 41 & 39 & $3.4 \mathrm{E} 022$ & $2^{20} 3 \cdot 5 \cdot 7 \ldots$ & 95 & 19 & $3.3 \mathrm{E} 008$ & $2^{11} 3^{2} 11 \cdot 13 \ldots$ & 387 & 28 & $6.8 \mathrm{E} 0142$ & $2^{17} 3^{3} 5 \cdot 7 \cdot 11^{2} 19^{2}$. \\
\hline 42 & 2 & 6 & $2 \cdot 3$ & 96 & 4 & 62 & $2 \ldots$ & 388 & 66 & $1.9 \mathrm{E} 041$ & $2^{23} 3^{5} 5^{4} 7^{2} 13 \cdot 17^{3}$ \\
\hline 43 & 16 & $4.5 \mathrm{E} 006$ & $2^{6} 3 \cdot 5 \ldots$ & 97 & 43 & $3.4 \mathrm{E} 023$ & $2^{8} 3^{9} 7^{2} 13 \cdot 19 \ldots$ & 389 & 296 & $4.9 \mathrm{E} 232$ & $2^{92} 3^{30} 5^{15} 7^{3} 11 \cdot 17^{3} 19^{6}$ \\
\hline 44 & 16 & $1.4 \mathrm{E} 007$ & $2^{11} 3 \cdot 19 \ldots$ & 98 & 3 & 6 & $2 \cdot 3$ & 390 & 12 & 389120 & $2^{12} 5 \cdot 19$ \\
\hline 45 & 16 & $8.2 \mathrm{E} 006$ & $2^{7} 3^{3} 7 \cdot 11 \ldots$ & 99 & 18 & $7.2 \mathrm{E} 007$ & $2^{10} 13 \ldots$ & 391 & 34 & $3.9 \mathrm{E} 018$ & $2^{15} 3^{2} 5 \cdot 7^{2} 11 \cdot 13 \ldots$ \\
\hline 46 & 10 & 19224 & $2^{3} 3^{3} \ldots$ & 100 & 20 & $1.3 \mathrm{E} 008$ & $2^{6} 3^{6} 7 \cdot 13$ & 392 & 20 & $2.9 \mathrm{E} 009$ & $2^{12} 3^{3} 19^{2}$ \\
\hline 47 & 32 & $3.8 \mathrm{E} 015$ & $2^{12} 3^{4} 7 \cdot 13 \ldots$ & 101 & 120 & $3.7 \mathrm{E} 079$ & $2^{45} 3^{13} 5^{3} 7^{5} 13^{2} 17^{2} 19^{2}$. & 393 & 205 & $9.1 \mathrm{E} 1532$ & $2^{65} 3^{13} 5^{12} 7^{10} 11^{3} 17^{2} 19^{2}$ \\
\hline 48 & 5 & 105 & $3 \cdot 5 \cdot 7$ & 102 & 35 & $1.7 \mathrm{E} 017$ & $2^{25} 3^{2} 5^{3} 7^{2} 19 \ldots$ & 394 & 47 & $2.8 \mathrm{E} 0282$ & $2^{10} 3^{8} 5 \cdot 7^{2} 13 \cdot 17 \ldots$ \\
\hline 49 & 13 & 92928 & $2^{8} 3 \cdot 11^{2}$ & 103 & 65 & $3.4 \mathrm{E} 040$ & $2^{24} 3^{9} 5^{2} 7^{3} 11 \cdot 19^{2} \ldots$ & 395 & 63 & $1.2 \mathrm{E} 0392$ & $2^{42} 3^{7} 7 \cdot 13 \cdot 19 \ldots$ \\
\hline 50 & 17 & $1.8 \mathrm{E} 007$ & $2^{9} 3^{4} 5 \cdot 7 \cdot 13$ & 104 & 10 & 6096 & $2^{4} 3 \ldots$ & 396 & 10 & 223202 & $2^{4} 3^{2} 5 \ldots$ \\
\hline 51 & 9 & 5120 & $2^{10} 5$ & 105 & 12 & 87552 & $2^{9} 3^{2} 19$ & 397 & 124 & $8.0 \mathrm{E} 0822$ & $2^{43} 3^{10} 5^{4} 7^{3} 13^{2} 19 \ldots$ \\
\hline 52 & 3 & 5 & & 106 & 54 & $3.7 \mathrm{E} 030$ & $2^{17} 3^{3} 5^{2} 7^{2} 11 \cdot 17^{3} 19 \ldots$ & 398 & 37 & $6.5 \mathrm{E} 0182$ & $2^{18} 3^{3} 5^{5} 19 \ldots$ \\
\hline 53 & 58 & $4.2 \mathrm{E} 033$ & $2^{20} 3^{4} 5^{4} 7^{4} 13 \cdot 17 \cdot 19 \ldots$ & 107 & 64 & $5.8 \mathrm{E} 036$ & $2^{17} 3^{8} 5^{3} 7 \cdot 11^{2} 13 \cdot 19 \ldots$ & 399 & 5 & 573 & $3 \cdot 19$ \\
\hline 54 & 11 & 100620 & $2^{2} 3^{2} 5 \cdot 13 \ldots$ & 108 & 13 & 491400 & $2^{3} 3^{3} 5^{2} 7 \cdot 13$ & 400 & 7 & 813 & \\
\hline
\end{tabular}

TABLE 2. Every $n \leq 1000$ is $(m, k)$-perfect for some $m, k$. This table shows, for $n \leq 108$ and $348 \leq n \leq 400$, the least such value of $m$, called $\widetilde{m}$, and the (approximate) corresponding value of $k$, called $\tilde{k}$. The prime factors of $\tilde{k}(n)$ less than 20 are also given. 
Proof. By definition, we have $\sigma^{\tilde{m}(n)}(n)=\tilde{k}(n) n$, so

$$
\begin{aligned}
\sigma^{\tilde{m}(n)-a}(t n) & =\sigma^{\tilde{m}(n)-a-M}\left(\sigma^{M}(t n)\right) \\
& =\sigma^{\tilde{m}(n)-a-M}\left(\sigma^{M+a}(n)\right) \\
& =\sigma^{\tilde{m}(n)}(n)=\frac{\tilde{k}(n)}{t} \cdot t n .
\end{aligned}
$$

This shows that $\widetilde{m}(t n) \leq \tilde{m}(n)-a$, and that if $\tilde{m}(t n)=\tilde{m}(n)-a$ then $\tilde{\tilde{k}}(t n)=\tilde{k}(n) / t$. Suppose $\widetilde{m}(t n)<\widetilde{m}(n)-a$. Then, by definition, $n \nmid \sigma^{j}(n)$ for $j=M+a, \ldots, \widetilde{m}(n)-1$, so, by $(3.1), t n \nmid \sigma^{j}(t n)$ for $j=M, \ldots, \widetilde{m}(n)-a$. Therefore, $\widetilde{m}(t n)<M$. Then

$$
\begin{aligned}
\tilde{k}(t n) & =\frac{\sigma^{\tilde{m}(t n)}(t n)}{t n} \\
& <\frac{\sigma^{M}(t n)}{t n}=\frac{\sigma^{M+a}(n)}{t n} \\
& =\frac{\sigma^{\tilde{m}(n)}(n)}{t n} \cdot \frac{\sigma^{M+a}(n)}{\sigma^{\tilde{m}(n)}(n)}=\alpha \frac{\tilde{k}(n)}{t} .
\end{aligned}
$$

Clearly, $\alpha<1$, completing the proof.

In fact, this number $\alpha$ would be expected to be quite small. For we have, extending (1.1),

$$
\sigma^{m}(n)=n \prod_{j=0}^{m-1} \sum_{d \mid \sigma^{j}(n)} \frac{1}{d} \quad \text { for } m \geq 1
$$

so that if $\sigma^{j}(n)$ is even for $j=M+a, \ldots, \widetilde{m}(n)$, then

$$
\begin{aligned}
\frac{\sigma^{\tilde{m}(n)}(n)}{\sigma^{M+a}(n)} & =\frac{\sigma^{\tilde{m}(n)-M-a}\left(\sigma^{M+a}(n)\right)}{\sigma^{M+a}(n)} \\
& \geq\left(1+\frac{1}{2}\right)^{\tilde{m}(n)-M-a} .
\end{aligned}
$$

Then $\alpha \leq\left(\frac{2}{3}\right)^{\tilde{m}(n)-M-a}$.

Many instances of Theorem 3.1 may be observed in Table 2. For example:
(a) $\sigma^{4}(5)=\sigma^{3}(10), \widetilde{m}(10)=\widetilde{m}(5)-1$ and $\tilde{k}(10)=$ $\frac{1}{2} \tilde{k}(5)$
(b) $\sigma^{3}(7)=\sigma(14), \tilde{m}(14)=\tilde{m}(7)-2$ and $\tilde{k}(14)=$ $\frac{1}{2} \tilde{k}(7)$
(c) $\sigma^{6}(9)=\sigma^{4}(36), \tilde{m}(36)=\widetilde{m}(9)-2$ and $\tilde{k}(36)=$ $\frac{1}{4} \tilde{k}(9)$;
(d) $\sigma^{4}(13)=\sigma(78), \widetilde{m}(78)=\tilde{m}(13)-3$ and $\tilde{k}(78)=$ $\frac{1}{6} \tilde{k}(13)$.

In each case, the other conditions of Theorem 3.1 must also be verified. It is easy to find solutions of (3.1), and we have done this for $n \leq 500, M+$ $a \leq 30$ and $t \leq 150$. There are a great many solutions, though not all satisfy the other conditions of the theorem. In all acceptable cases, we confirmed that, in the notation of the theorem, $\widetilde{m}(t n)=\widetilde{m}(n)-a$. Here are some of those examples, giving extensions of Table 2:
(e) $\sigma^{10}(101)=\sigma^{6}(2020), \widetilde{m}(2020)=\widetilde{m}(101)-4$ and $\tilde{k}(2020)=\frac{1}{20} \tilde{k}(101)$
(f) $\sigma^{10}(233)=\sigma^{8}(2330), \widetilde{m}(2330)=\widetilde{m}(233)-2$ and $\tilde{k}(2330)=\frac{1}{10} \tilde{k}(233)$
(g) $\sigma^{11}(394)=\sigma^{10}(6698), \widetilde{m}(6698)=\widetilde{m}(394)-1$ and $\tilde{k}(6698)=\frac{1}{17} \tilde{k}(394)$
(h) $\sigma^{8}(197)=\sigma^{2}(29550), \widetilde{m}(29550)=\widetilde{m}(197)-6$ and $\tilde{k}(29550)=\frac{1}{150} \tilde{k}(197)$.

In (g), for example, where $6698=17 \cdot 394$, it is clear that we need to know at least the small prime factors of $\tilde{k}(n)$ for each $n$ in order that the condition $t \mid \tilde{k}(n)$ might be checked. These small prime factors, namely those less than 20 , have been included in Table 2.

There is no reason, in (3.1), why $a$ cannot in fact be zero or negative (provided $M+a>0$ ). We found one instance of this in the above search: $\sigma^{8}(404)=\sigma^{8}(808)$, from which, as in Theorem 3.1, we could verify that $\widetilde{m}(808)=\widetilde{m}(404)$ and

$$
\tilde{k}(808)=\frac{1}{2} \tilde{k}(404) \text {. }
$$

This led us to seek solutions of the equation

$$
\sigma^{m}(t n)=\sigma^{m}(n)
$$

over a much larger range. For $t \leq 4, m \leq 12$ and $n \leq 10^{5}$, the solutions are listed in Table 3 . Note that for any pair $\left(m_{0}, n\right)$ that satisfies (3.2) for some $t$, we also have the solutions $(m, n)$ for all $m \geq m_{0}$.

Following on from this, can it be proved that the equation $\sigma(\sigma(2 n))=\sigma(\sigma(n))$ has no solutions? 


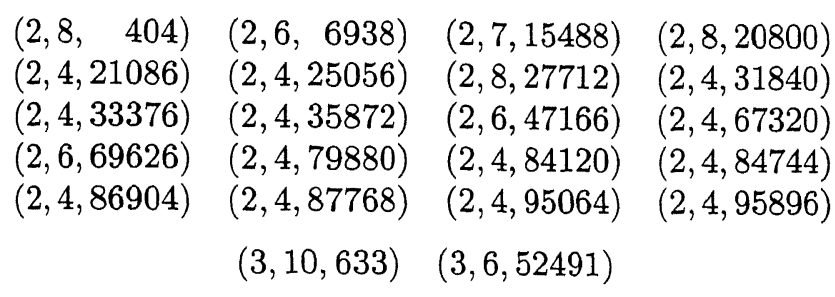

TABLE 3. Solutions $(t, m, n)$ of $\sigma^{m}(t n)=\sigma^{m}(n)$ with $t \leq 4, m \leq 12$, and $n \leq 10^{5}$.

We can prove only that for any $n$ satisfying this equation we must have $2^{a} \| n$, with $\sigma\left(2^{a+1}-1\right) \geq$ $2^{a+2}$. This condition is satisfied by $a=11,23,35$, $39,47, \ldots$ For these five smallest possible values of $a$, we have checked each $n=2^{a} l$, with $l<10^{4}$ odd, and found no solutions.

\section{DISCUSSION OF THE SIX STATEMENTS}

The preceding section has been largely concerned with statement (iv) of the six by [Erdös et al. 1990] given in the Introduction. This was also posed by Carl Pomerance as unsolved problem 94:13 at the Western Number Theory Conference in December 1994 at San Diego. The following slightly edited comment accompanied the problem: "It is inconceivable that the conjecture is false. Each (odd part of) $n$ divides $2^{r s}-1$ for a suitable $s$ and all $r$, and $\sigma\left(2^{r s-1}\right)=2^{r s}-1$. As $m$ increases, $\sigma^{m}(n)$ increases quite rapidly, and so does the power of 2 it contains, albeit very erratically. How can the sequence of exponents of 2 avoid all members of the arithmetic progression $r s-1$ ?"

We observe next that Theorem 3.1 shows some relationship between statements (iv) and (vi) in the Introduction, in that a value for $m$ for which $t n \mid \sigma^{m}(t n)$ may be inferred from a suitable solution of $\sigma^{m_{1}}(n)=\sigma^{m_{2}}(t n)$. If we write $n_{1}, n_{2}$, for $n, t n$, respectively, in Theorem 3.1 then clearly we have

$$
n_{1} \tilde{k}\left(n_{1}\right)=n_{2} \tilde{k}\left(n_{2}\right)
$$

Furthermore, given $n_{1}$ and $n_{2}$, if we notice that (4.1) is satisfied then we have a solution of the equation $\sigma^{m_{1}}\left(n_{1}\right)=\sigma^{m_{2}}\left(n_{2}\right)$, namely $m_{1}=\widetilde{m}\left(n_{1}\right)$ and $m_{2}=\tilde{m}\left(n_{2}\right)$. This demonstrates a relationship between the two statements in the reverse direction. We have observed from Table 2 the following nine instances of pairs $\left(n_{1}, n_{2}\right)$ that satisfy (4.1), but in which $n_{2}$ is not a multiple of $n_{1}$ : $(7,24),(9,168),(10,12),(14,24),(18,120)$, $(36,168),(62,96),(72,336)$ and $(341,384)$.

While Table 2 and the further computations for $n \leq 1000$ support the truth of statement (iv), we do not believe that statement (vi) is true. The figure below shows how sequences $\left\{\sigma^{i}(n)\right\}_{i=1}^{\infty}$, for any $n$ in the figure, merge into the sequence 480,1512 , $4800, \ldots$ (For example, $\sigma(45)=78, \sigma^{2}(45)=168$, $\left.\sigma^{3}(45)=480, \ldots\right)$

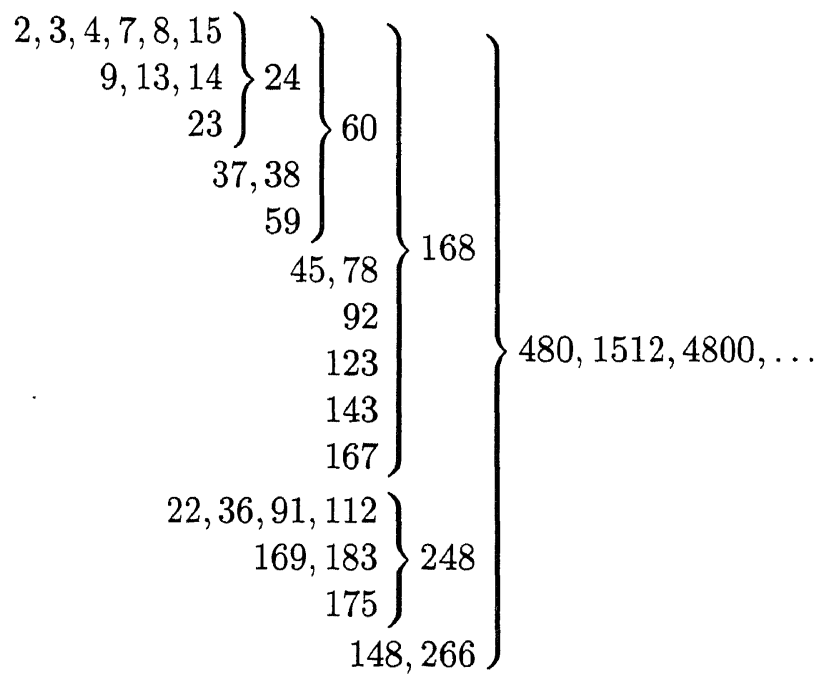

No other values of $n \leq 200$ are such that the sequence $\left\{\sigma^{i}(n)\right\}$ intersects (and joins with) any of those in the figure, for values of $\sigma^{i}(n)<10^{200}$. Three parameters determine the numbers in this figure: we call it a $\left(\pi_{1}, \pi_{2}, \pi_{3}\right)$-tree, with $\pi_{1}$ the smallest number in the tree, and $\pi_{2}, \pi_{3}$ such that all sequences $\left\{\sigma^{i}(n)\right\}$ with $\pi_{1} \leq n \leq \pi_{2}$ and $\sigma^{i}(n)<$ $\pi_{3}$ have nonempty intersection with $\left\{\sigma^{i}\left(\pi_{1}\right)\right\}$. If we first specify $\pi_{2}$ and $\pi_{3}$ (200 and $10^{200}$, here) then we may determine successive $\left(\pi_{1}, \pi_{2}, \pi_{3}\right)$-trees for all $\pi_{1} \leq \pi_{2}$. There are $21\left(\pi_{1}, 200,10^{200}\right)$-trees, having the following values of $\pi_{1}$ :

$2,5,16,19,27,29,33,49,50,52,66,81$, $85,105,146,147,163,170,189,197,199$. 
The approach here was as follows. We calculated the sequences $\left\{\sigma^{i}(n)\right\}$ for each $n, 2 \leq n \leq 200$, and determined which sequences were such that the first term exceeding $10^{10}$ equalled such a term from an earlier sequence. There were $21\left(\pi_{1}, 200,10^{10}\right)$ trees obtained this way, and these were tested further for intersection by determining the values of the first terms that exceeded $10^{200}$. The trees remained distinct, and we conjecture that this will stay true as $\pi_{3} \rightarrow \infty$.

We also found $64\left(\pi_{1}, 1000,10^{100}\right)$-trees.

Some evidence for statement (iii) in the Introduction is provided by the further computations that extend those for Table 2. The following is the list of those $N<1000$ for which $\widetilde{m}(n)<\widetilde{m}(N)$ for all $n<N$. (We called such numbers $N$ megaperfect in a talk at CANT'95, the Computational Algebra and Number Theory conference held at Macquarie University, Sydney, in April 1995.)

$\begin{array}{rrrrrrrr}N & 1 & 2 & 3 & 5 & 9 & 11 & 23 \\ \widetilde{m}(N) & 1 & 2 & 4 & 5 & 7 & 15 & 16 \\ N & 25 & 29 & 59 & 67 & 101 & 131 & 173 \\ \tilde{m}(N) & 17 & 78 & 97 & 101 & 120 & 174 & 214 \\ N & 202 & 239 & 353 & 389 & 401 & 461 & 659 \\ \tilde{m}(N) & 239 & 261 & 263 & 296 & 380 & 557 & 1287\end{array}$

We set

$$
h(n)=\frac{\left(\sigma^{\tilde{m}(n)}(n)\right)^{1 / \widetilde{m}(n)}}{\log \tilde{m}(n)} .
$$

For the last three values of $N$ above, we have

$$
\begin{array}{rccc}
n & 401 & 461 & 659 \\
h(n) & 1.1146 & 1.1276 & 1.1658
\end{array}
$$

which suggests that $\left(\sigma^{m}(n)\right)^{1 / m}$ is at least of the same order as $\log m$, as $m \rightarrow \infty$, for any $n$.

With regard to $\widetilde{m}(659)$, we remark that

$$
\begin{aligned}
\tilde{k}(659) & =2^{276} 3^{100} 5^{44} 7^{28} 11^{21} 13^{14} 17^{14} 19^{8} \ldots \\
& \approx 1.5 \cdot 10^{1183} .
\end{aligned}
$$

In the calculation of $\tilde{m}(659)$, we had to factorise a difficult 104-digit composite factor of $\sigma^{1240}(659)$. This number, which we indicate by $\mathrm{C} 104$, arose as follows. We found that $2^{372} \| \sigma^{1238}(659)$, so that $\sigma\left(2^{372}\right)=\left(2^{373}-1\right) \mid \sigma^{1239}(659)$. Now, $2^{373}-1=$
$25569151 \cdot \mathrm{P} 105$, where $\mathrm{P} 105$ is a prime number of 105 decimal digits. Consequently, $\sigma(\mathrm{P} 105)=$ $(\mathrm{P} 105+1) \mid \sigma^{1240}(659)$ and $\mathrm{P} 105+1=2 \cdot 7 \cdot \mathrm{C} 104$. We were unable to factorise this $\mathrm{C} 104$ with the elliptic curve method or with the quadratic sieve method, and therefore asked Peter Montgomery's help, noticing that

$$
\mathrm{C} 104=\frac{2^{373}-1+25569151}{2 \cdot 7 \cdot 25569151} .
$$

Peter constructed the two polynomials

$p_{1}(x)=5 x-2^{74}, \quad p_{2}(x)=500 x^{5}+\frac{25569151-1}{50}$,

which have the property that

$p_{1}(m) \equiv p_{2}(m) \equiv 0(\bmod C 104) \quad$ for $m=2^{74} 5^{-1}$.

This enabled him to apply the Special Number Field Sieve method [Lenstra and Lenstra 1993] and factorise C104 within two days on SGI workstations at CWI Amsterdam and the Cray C90 at SARA Amsterdam, into the product of 38-digit and 67-digit primes:

$$
\begin{gathered}
\mathrm{C} 104=18223164902649732703974292810329988561 \\
\times 294930871353255542584246554605934608110- \\
4682577291637010561295300423 .
\end{gathered}
$$

We also used the $21\left(n, 200,10^{200}\right)$-trees, with $n=\pi_{1}$ in (4.2), to investigate statements (i), (ii) and (iii). The results are summarised in Table 4. We remark that if statement (iii) is true and the sequence $\left\{\left(\sigma^{i}(n)\right)^{1 / i}\right\}$ is eventually monotone, then (ii) is true, since $\left(\sigma^{i+1}(n)\right)^{1 /(1+i)}>\left(\sigma^{i}(n)\right)^{1 / i} \mathrm{im}$ plies

$$
\frac{\sigma^{i+1}(n)}{\sigma^{i}(n)}>\left(\sigma^{i}(n)\right)^{1 / i}
$$

Our computations strongly suggest that indeed the sequence $\left\{\left(\sigma^{i}(n)\right)^{1 / i}\right\}$ is eventually monotone, for every $n$.

We turn finally to statement (v). As evidence in favour of this statement, we showed that every number up to 400 occurs as a divisor in the sequence $\left\{\sigma^{i}(n)\right\}$, for each of the 21 values of $n$ in (4.2). The results are summarised in Table 5. 


\begin{tabular}{|r|cccc|cccc|}
\hline$n$ & $j_{1}$ & $\alpha_{1}$ & $\beta_{1}$ & $\beta_{1} / \log j_{1}$ & $j_{2}$ & $\alpha_{2}$ & $\beta_{2}$ & $\beta_{2} / \log j_{2}$ \\
\hline 2 & 146 & 6.2437 & 4.8927 & 0.98176 & 263 & 7.8129 & 5.7938 & 1.03978 \\
5 & 144 & 6.8248 & 4.9610 & 0.99822 & 262 & 7.3602 & 5.8341 & 1.04773 \\
16 & 143 & 6.3581 & 5.0681 & 1.02120 & 260 & 7.2318 & 5.9191 & 1.06445 \\
19 & 140 & 6.2237 & 5.2215 & 1.05663 & 257 & 7.4125 & 6.0250 & 1.08576 \\
27 & 138 & 6.6011 & 5.3063 & 1.07692 & 256 & 7.4307 & 6.0797 & 1.09640 \\
29 & 143 & 6.9807 & 5.0686 & 1.02131 & 260 & 7.3834 & 5.9227 & 1.06511 \\
33 & 142 & 6.3337 & 5.1231 & 1.03375 & 259 & 7.6907 & 5.9330 & 1.06770 \\
49 & 142 & 6.8223 & 5.0856 & 1.02619 & 260 & 7.3791 & 5.9128 & 1.06332 \\
50 & 141 & 7.1219 & 5.1384 & 1.03831 & 258 & 7.7576 & 5.9640 & 1.07403 \\
52 & 140 & 6.3248 & 5.2049 & 1.05328 & 257 & 8.3219 & 6.0347 & 1.08752 \\
66 & 139 & 6.4359 & 5.2554 & 1.06504 & 255 & 7.4043 & 6.0885 & 1.09876 \\
81 & 140 & 6.9101 & 5.1895 & 1.05016 & 257 & 8.1663 & 6.0044 & 1.08205 \\
85 & 143 & 6.7800 & 5.0216 & 1.01183 & 260 & 7.8790 & 5.8813 & 1.05765 \\
105 & 141 & 7.0380 & 5.1771 & 1.04614 & 258 & 7.9647 & 5.9891 & 1.07854 \\
146 & 138 & 6.0071 & 5.3216 & 1.08003 & 255 & 7.1539 & 6.1125 & 1.10309 \\
147 & 139 & 6.6003 & 5.2756 & 1.06914 & 256 & 8.1533 & 6.0440 & 1.08996 \\
163 & 139 & 7.1172 & 5.2817 & 1.07037 & 256 & 7.4892 & 6.0688 & 1.09443 \\
170 & 138 & 6.8193 & 5.3547 & 1.08675 & 255 & 7.7101 & 6.1182 & 1.10411 \\
189 & 138 & 6.9452 & 5.3358 & 1.08291 & 256 & 8.1988 & 6.0853 & 1.09741 \\
197 & 139 & 6.6808 & 5.2831 & 1.07065 & 256 & 8.0667 & 6.0462 & 1.09036 \\
199 & 139 & 5.9943 & 5.2720 & 1.06841 & 256 & 7.5814 & 6.0618 & 1.09317 \\
\hline
\end{tabular}

TABLE 4. For each $n, j_{1}$ is the smallest value of $i$ such that $\sigma^{i}(n)>10^{100}, j_{2}$ is the smallest value of $i$ such that $\sigma^{i}(n)>10^{200}$, and $\alpha_{u}=\sigma^{j_{u}+1}(n) / \sigma^{j_{u}}(n), \beta_{u}=\left(\sigma^{j_{u}}(n)\right)^{1 / j_{u}}$, for $u=1,2$.

\begin{tabular}{|c|c|c|c|c|c|c|c|c|c|}
\hline$n$ & $j_{2}$ & 239 & 283 & 293 & $\begin{array}{c}\text { hard" } \\
347\end{array}$ & $\begin{array}{c}\text { isors } d \\
353\end{array}$ & 359 & 383 & 389 \\
\hline 2 & 263 & 290 & & & & & & & $370 *$ \\
\hline 5 & 262 & 290 & & & & 275 & & & $293 *$ \\
\hline 16 & 260 & $346 *$ & & & & 274 & 265 & & 296 \\
\hline 19 & 257 & $307 *$ & & & & & & & 295 \\
\hline 27 & 256 & $295 *$ & & 287 & & & & & \\
\hline 29 & 260 & 287 & 271 & & & & 262 & & $301 *$ \\
\hline 33 & 259 & $322 *$ & & & & & & & \\
\hline 49 & 260 & & & 295 & & & & & $299 *$ \\
\hline 50 & 258 & & 261 & & & $268 *$ & & & 265 \\
\hline 52 & 257 & $323 *$ & & & & & & & 297 \\
\hline 66 & 255 & 263 & & & & & & & $285 *$ \\
\hline 81 & 257 & $352 *$ & & & & 307 & & 271 & 278 \\
\hline 85 & 260 & & & & & $264 *$ & & & 262 \\
\hline 105 & 258 & $281 *$ & & & & & & & \\
\hline 146 & 255 & 281 & & & $316 *$ & & & & 263 \\
\hline 147 & 256 & 300 & & & & 285 & & 293 & $328 *$ \\
\hline 163 & 256 & & & & & & & & $266 *$ \\
\hline 170 & 255 & & & $283 *$ & & & & & \\
\hline 189 & 256 & 303 & & & & & & 282 & $305 *$ \\
\hline 197 & 256 & & & 285 & & 257 & & & $289 *$ \\
\hline 199 & 256 & $292 *$ & & & & & & & 276 \\
\hline
\end{tabular}

TABLE 5. Smallest value of $i$ for which $d \mid \sigma^{i}(n)$, for given $d$ (see top of next page for full explanation). 
We give in that table the "hard" divisors, those that did not divide any term of $\left\{\sigma^{i}(n)\right\}$ for some $n$ in (4.2) and $i \leq j_{2}$, with $j_{2}$ as in Table 4; and, for each such divisor $d$, we give the first index $i>j_{2}$ for which $d \mid \sigma^{i}(n)$. The largest such index for each $n$ is marked by ${ }^{*}$, so every number up to 400 divides a term of this sequence for some value of $i$ up to the marked value.

For example, all positive integers less than or equal to 400 , except 239 and 389 , divide a term of the sequence $\left\{\sigma^{i}(2)\right\}$ for some value of $i$ with $0 \leq$ $i \leq j_{2}$, where $j_{2}=263$ is the index of the first term in this sequence that exceeds $10^{200}$; furthermore, $239 \mid \sigma^{290}(2)$ and $389 \mid \sigma^{370}(2)$.

Not surprisingly, the larger megaperfect numbers less than 400 are in the list of hard divisors.

\section{ACKNOWLEDGMENTS}

Part of Table 2 was computed independently by Robert Harley. In particular, he computed $\widetilde{m}(n)$ and $\tilde{k}(n)$ for $n \leq 658$, and the $\sigma^{i}(659)$-sequence up to $i=1035$.

We are grateful to Peter Hagis, Jr, for carefully reading an early version of this paper and to Peter Montgomery for his help with the difficult factorisation described above.

Part of this paper was written while the second author was a Visiting Professor at the University of Technology, Sydney in July-November 1995. He thanks the first author and UTS for the warm hospitality and the stimulating working conditions.

\section{REFERENCES}

[Cohen and te Riele 1995] G. L. Cohen and H. J. J. te Riele, "Iterating the sum-of-divisors func- tion", Research Report R95-10, School of Mathematical Sciences, University of Technology, Sydney, 1995; CWI Report NM-R9525, CWI Amsterdam, 1995, available at ftp://ftp.cwi.nl/pub/CWIreports/ NW/NM-R9525.ps.Z.

[Erdős 1955] P. Erdős, "On amicable numbers", Publ. Math. Debrecen 4 (1955), 108-111.

[Erdős et al. 1992] P. Erdős, A. Granville, C. Pomerance, and C. Spiro, "On the normal behavior of the iterates of some arithmetical functions", pp. 165204 in Analytic Number Theory, Allerton Park, 1989, Birkhaüser, Boston, 1990.

[Guy 1994] R. K. Guy, Unsolved Problems in Number Theory, 2nd. ed., Springer, New York, 1994.

[Hausman 1982] M. Hausman, "The solution of a special arithmetic equation", Canad. Math. Bull. 25 (1982), 114-117.

[Kanold 1969] H.-J. Kanold, "Über 'Super perfect numbers'", Elem. Math. 24 (1969), 61-62.

[Lenstra and Lenstra 1993] A. K. Lenstra and H. W. Lenstra, Jr., The Development of the Number Field Sieve, Lecture Notes in Mathematics 1554, Springer, Berlin, 1993.

[Lord 1975] G. Lord, "Even perfect and super perfect numbers", Elem. Math. 30 (1975), 87-88.

[Maier 1984] H. Maier, "On the third iterates of the $\varphi$ and $\sigma$-functions", Colloq. Math. 49 (1984), 123-130.

[Pomerance 1975] C. Pomerance, "On multiply perfect numbers with a special property", Pacific J. Math. 57 (1975), 511-517.

[Schroeppel 1993] R. Schroeppel, "1385 multiperfect numbers", 4 May 1993, available with updates from the author at rcs@cs.arizona.edu. In private communication, there were 2125 multiperfect numbers listed in January 1996.

[Suryanarayana 1969] D. Suryanarayana, "Super perfect numbers", Elem. Math. 24 (1969), 16-17.

Graeme L. Cohen, School of Mathematical Sciences, University of Technology, Sydney, PO Box 123, Broadway, NSW 2007, Australia (glc@maths.uts.edu.au)

Herman J. J. te Riele, CWI, Department of Numerical Mathematics, Kruislaan 413, 1098 SJ Amsterdam, The Netherlands (herman.te.riele@cwi.nl) 


\section{Errata to}

\section{Iterating the Sum-of-Divisors Map}

Graeme L. Cohen and Herman J. J. te Riele

Volume 5 (1996), pages 91-100

In Table 1 on page 93 , the $(2,15)$-perfect number $506967552=2^{9} \cdot 3^{3} .7 .13^{2} .31$ is missing, due to an error made while we edited an output file. We thank Jan Munch Pedersen of Vejle Business College in Vejle, Denmark for this remark.

Graeme L. Cohen, School of Mathematical Sciences, University of Technology, Sydney, PO Box 123, Broadway, NSW 2007, Australia (glc@maths.uts.edu.au)

Herman J. J. te Riele, CWI, Department of Numerical Mathematics, Kruislaan 413, 1098 SJ Amsterdam, The Netherlands (herman.te.riele@cwi.nl) 